
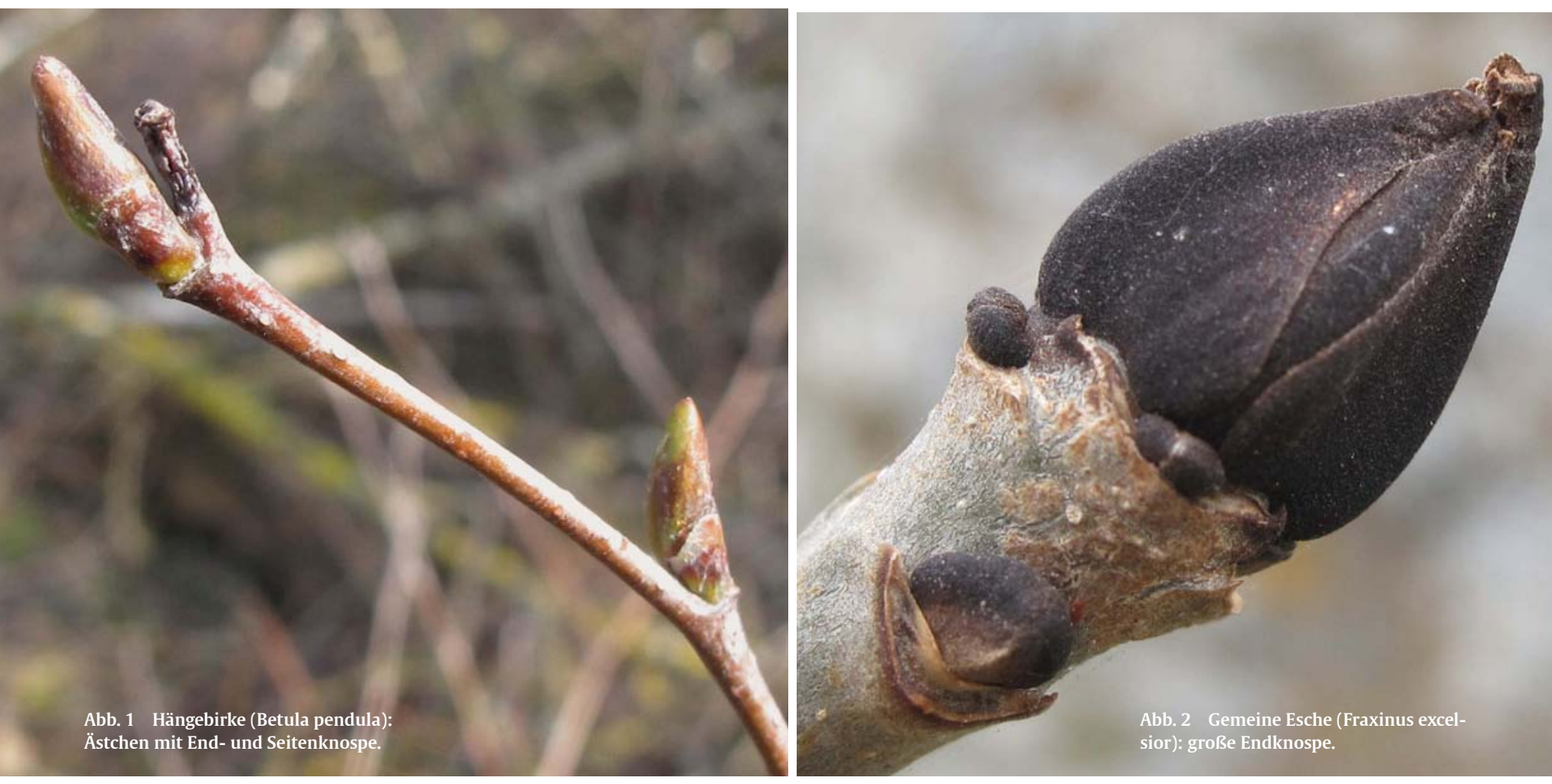

\title{
Von Diurese bis
}

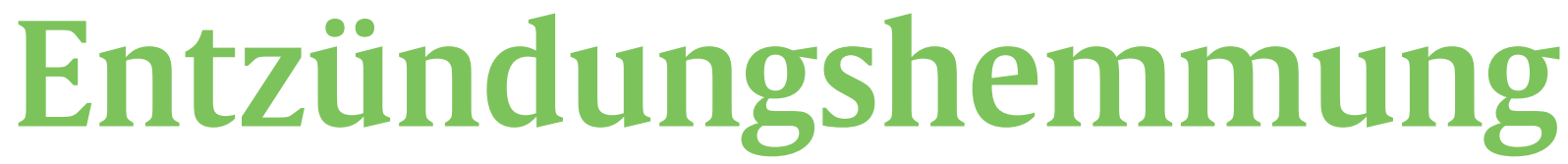

\section{Die führenden Nierenmittel der GEMIMOTHERAPIE \\ Cornelia Stern}

SIE KÖNNEN bei vielen Beschwerden eingesetzt werden, so auch auf der Ebene der Harnwege: Gemmopräparate verfügen über besondere entgiftende Fähigkeiten und stehen damit in engem Bezug zur Niere als einem (neben der Leber) der beiden großen Ausleitungsorgane. Die Kombination mit schulmedizinischen Medikamenten ist hierbei problemlos möglich, Wechselwirkungen wurden bislang nicht dokumentiert.

\section{Birke: ideal auch bei Ödemen und Niereninsuffizienz}

Die Hängebirke (Betula pendula) gehört in der Phytotherapie zu den großen Nierenpflanzen, deren Blätter mit ihren Flavonoiden und Saponinen für eine Durch- spülung der Harnwege sorgen. Auch in der Gemmotherapie findet mit den Birkenknospen die Entgiftung und Ausleitung über die Nieren statt. Laut Untersuchungen - insbesondere von Dr. Pol Henry und Dr. Max Tétau - jedoch auch über die Leber.

\section{- AUF EINEN BLICK}

\section{Indikationen für Birken-Gemmopräparate}

- Steigerung der Diurese bei leichter Niereninsuffizienz

- Durchspülung der Harnwege bei Blasenentzündung, in Kombination mit dem antientzündlich wirkenden Gemmo Ribes nigrum (Schwarze Johannisbeere) sowie antibakteriell wirkenden Senfölglykosid-Drogen der Phytotherapie wie Kapuzinerkresse und Meerrettich (zum Beispiel Angocin ${ }^{\circledR}$ Anti-Infekt, Fa. Repha)

- verstärkte Ausleitung der Harnsäure bei gichtanfälligen Patienten (beim akuten Gichtanfall ist Gemmo Fraxinus besser geeignet)

- Steigerung der Diurese und damit Prophylaxe von Nierengrieß und Nierensteinen 
In kleinen Studien wurde die Diuresekapazität mit und ohne Anwendung von Gemmo Birke untersucht. Dabei stellte sich heraus, dass die Nieren mit der Unterstützung durch das Knospenmittel eine fünfmal stärkere Diurese leisteten und dies ohne eine zusätzliche Trinkmenge von 2-31 Wasser. Daher kann die Birke als Knospenmazerat im Unterschied zum Birkentee ödematöse Wasseransammlungen im Körper durch therapeutischen Wasserentzug ausschwemmen und auch bei Patienten mit einer Nieren- oder Herzinsuffizienz (die selbst häufig Ödeme verursachen) eingesetzt werden. Denn beide Organe müssen so keine zusätzliche Wasseraufnahme verkraften. Die vermehrte Entgiftung und Ausleitung verbessert auch rheumatische Erkrankungen, Ekzeme und andere Hautausschläge und führt zu einer gesteigerten Ausleitung harnpflichtiger Substanzen.

Das Gemmopräparat Birke kann man über mehrere Wochen einnehmen (Standarddosierung: morgens drei Sprühstöße in den Mund). Es wirkt milder als andere Nieren-Gemmomittel und ist vor allem für Beschwerden angezeigt, die sich selbst ebenfalls noch „milde“ zeigen. So hilft es unter anderem zuverlässig bei den ersten Anzeichen von (rheumatischen) Gelenkschmerzen wie morgendlicher Gelenksteifigkeit und abnehmender Beweglichkeit.

Birkenknospen gelten als Mittel der Jugendlichkeit und Leichtigkeit. Sie haben die Fähigkeit, den Körper durch ihre ausleitende und damit entgiftende Wirkung gesundzuerhalten oder sogar zu „verjüngen“. Gerne spricht man von einem Anti-Aging-Effekt.

\section{Esche bei schwereren Nieren- störungen und Gichtanfall}

Die Esche (Fraxinus excelsior) nimmt in der heutigen Phytotherapie nur einen geringen Stellenwert ein. Einzig die Eschenblätter verfügen über eine HMPC-Monografie als traditionelles pflanzliches Arzneimittel. Diese nennt die Indikationen „leichte Gelenkschmerzen“ und „zur

\author{
KURZ GEFASST \\ 1 Die Gemmotherapie nutzt das junge, teilungsaktive Pflanzen- \\ gewebe der Knospen und verfügt damit über wertvolle Inhalts- \\ stoffe, die in fertig ausgebildeten Pflanzenteilen nicht vorkom- \\ men. \\ 2 Zu den wichtigsten Nierenmitteln der Gemmotherapie zählen \\ Birke, Esche, Pappel, Wacholder und Preiselbeere, die insbesonde- \\ re als Diuretika und Antiphlogistika wirken. \\ $3 \begin{aligned} & \text { Knospenpräparate haben sich insbesondere bei akuten oder rezi- } \\ & \text { divierenden Blasenentzündungen sowie begleitend bei Nierenstö- }\end{aligned}$ \\ rungen und Gicht bewährt.
}

Durchspülung der Harnwege bei Blasenentzündung“. Beide Einsatzgebiete deuten auf eine Steigerung der Entgiftung und Ausleitung über die Nieren hin.

Im Vergleich zur Birke liegt der Schwerpunkt in der Gemmotherapie hier jedoch auf der begleitenden Behandlung schwererer klinischer Beschwerdebilder (Dosierung: 2- bis 3-mal tgl. 2-3 Sprühstöße in den Mund). Auffallend ist die Tendenz zu „Ablagerungserkrankungen“ der Niere (Nierengrieß, Nierensteine) wie auch der Gelenke (Gicht). Aufgrund ihrer entzündungshemmenden Wirkung wird die Esche jedoch auch gerne bei Arthritis oder in der entzündlichen Phase einer Arthrose verwendet. Typische Zeichen dafür sind geschwollene, rote und heiße Gelenke.

Die diuretische Wirkung der Eschenknospen auf die Harnwege geht auch mit einer gesteigerten Ausleitung harnpflichtiger Substanzen einher und verhindert Auskristallisationen in der Niere.

Das Gemmopräparat Esche kann über einen längeren Zeitraum eingenommen werden. In den akut-entzündlichen Phasen einer Erkrankung ist die Esche jedoch besonders wirksam. Zudem ist sie das Mittel der Wahl, wenn sich die Beschwerden bei kaltem und/oder nassem Wetter verschlechtern.

\section{Entzündungshemmend und schmerzlindernd: Pappel}

Die Schwarzpappel (Populus nigra) ist in der modernen Phytotherapie wenig gebräuchlich. Dafür blickt sie auf eine lan-

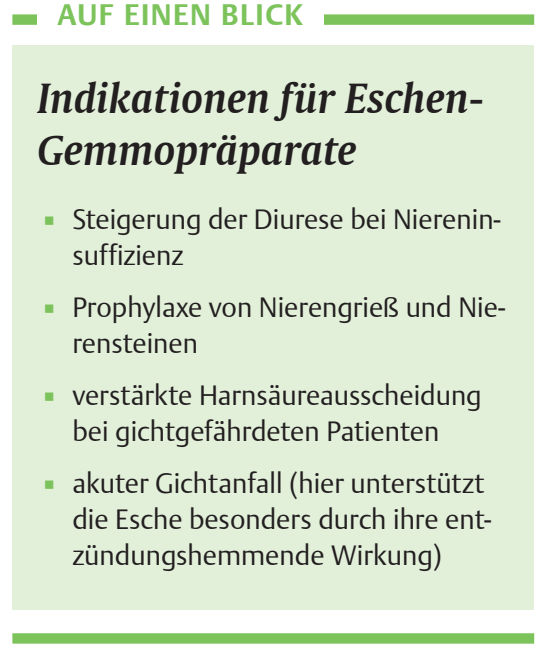

ge Tradition in der Volksheilkunde zurück. Diese findet sich im Wirkstoffprofil bestätigt, denn die enthaltenen Salicylate werden im menschlichen Stoffwechsel zu Salicylsäure metabolisiert. Diese wirkt analgetisch, fiebersenkend und entzündungshemmend.

Die länglich-spitzen Pappelknospen sind von einem klebrigen Knospenleim umhüllt und verströmen einen warmen, balsamischen Duft. Honigbienen sammeln gerne diese klebrige Substanz, vermischen sie mit ihrem Speichel, kleiden damit den Bienenstock aus und schützen ihr Zuhause auf diese Weise vor Bakterien, Viren und Pilzen. Wir kennen dieses pflanzlich-tierische Gemisch als Propolis, das den Körper bei viralen und bakteriellen Erkältungskrankheiten unterstützt.

In der Gemmotherapie gehört die Pappel ebenfalls zu den Nierenmitteln. 


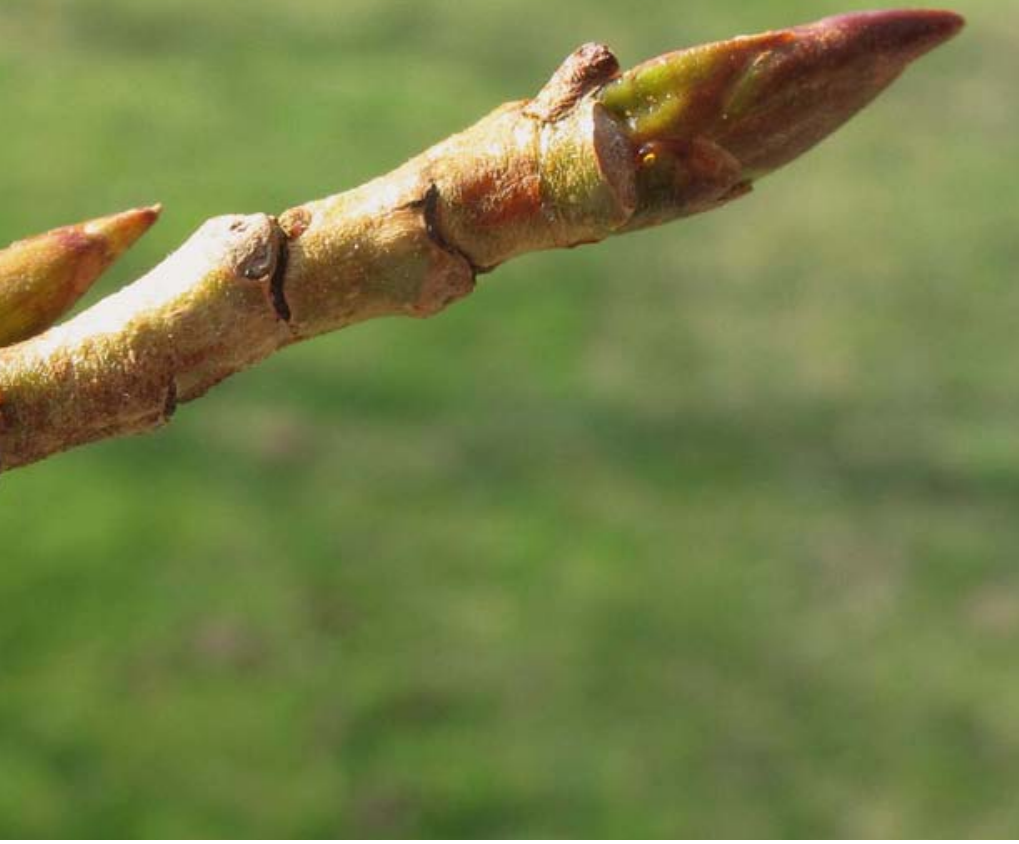

Abb. 3 Schwarzpappel (Populus nigra): Endknospe mit mehreren dreispurigen Blattnarben.

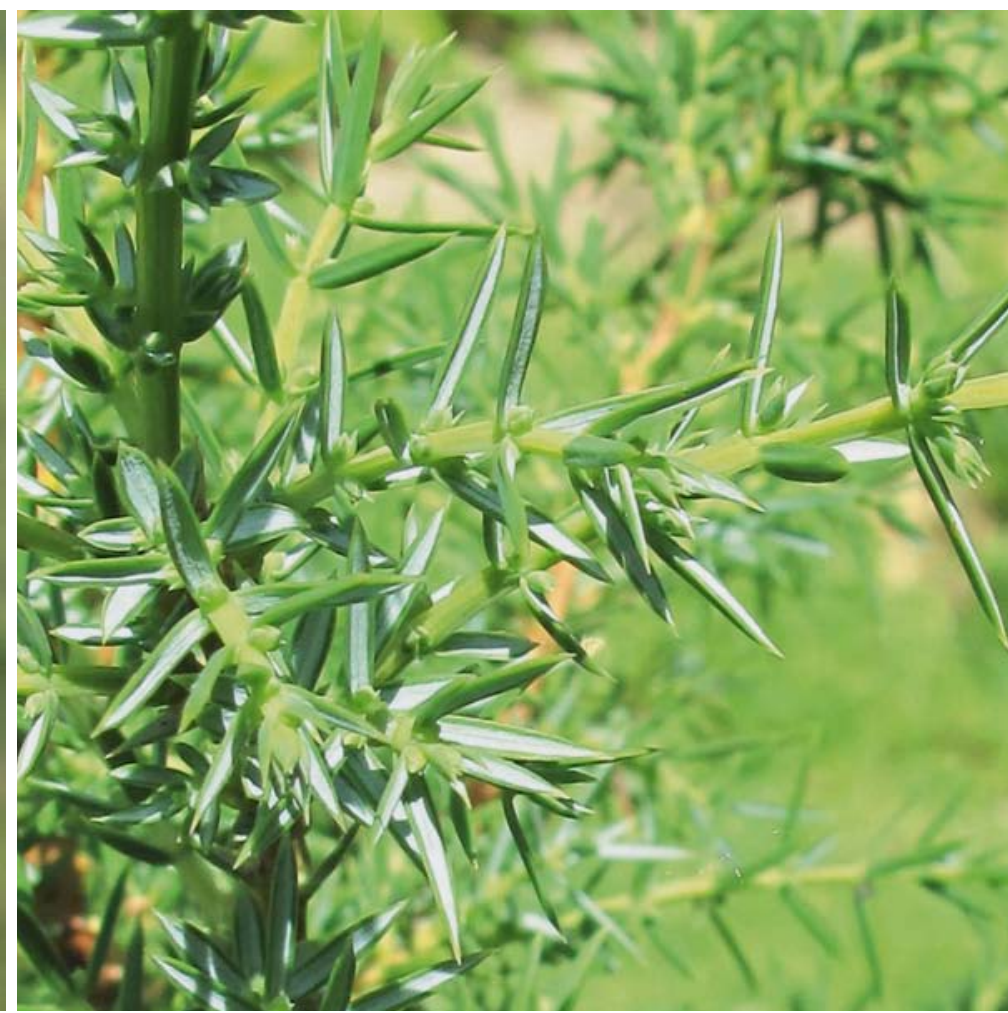

Abb. 4 Gemeiner Wacholder (Juniperus communis): Endknospen und Seitenknospen eines Wacholdertriebs.
Die Wirkung der Knospen auf die Harnwege beruht einerseits auf der Entzündungshemmung und Schmerzlinderung, andererseits auf ihrer antibakteriellen Wirkung. Der Therapieschwerpunkt liegt hier vor allem auf Beschwerden, bei denen sich etwas nicht im Fluss befindet. Dazu gehören unter anderem arterielle Durchblutungsstörungen wie das Raynaud-Syndrom und die Arteriosklerose. Harnsäure kristallisiert insbesondere in Gewebsflüssigkeiten aus, in denen keine oder kaum Fließbewegung

- AUF EINEN BLICK

\section{Indikationen für Pappel-Gemmopräparate}

- akute Blasenentzündung

- chronisch rezidivierende Blasenentzündungen

- Reizblase

- interstitielle Zystitis

- Gichtanfall (entzündungshemmend und schmerzlindernd) stattfindet. Dies betrifft unter anderem die Gelenkflüssigkeit der Synovialschleimhaut - im übertragenen Sinn auch ein Thema des „im Fluss bleibens“. So gehört auch die Gicht, insbesondere der Gichtanfall, zu den Indikationen der Pappel. Genauso kann man die Reizblase wie die interstitielle Zystitis als Hindernis für den Harnfluss ansehen: Die Blase ist gereizt, und es besteht mehr oder weniger heftiger Harndrang, ohne dass jedoch entsprechende Urinmengen zur Ausscheidung gelangen.

Das Gemmopräparat Pappel kann wie die zuvor beschriebenen Mittel mit einer Dosierung von 2- bis 3-mal tgl. 2-3 Sprühstößen über einen längeren Zeitraum verordnet werden. In akut-entzündlichen, schmerzhaften Phasen oder wenn Bakterien beteiligt sind, sollte die Dosierung erhöht werden auf bis zu stündlich 2-3 Sprühstößen.

\section{Wacholder als potentes pflanzliches Nierenmittel}

Der Gemeine Wacholder (Juniperus communis) behauptet als Nieren- und
- AUF EINEN BLICK

\section{Indikationen für Wachol- der-Gemmopräparate}

- unterstützend bei Nephritis

- unterstützend bei Pyelonephritis

- chronisch-rezidivierende Blasenentzündungen

- unterstützend bei Niereninsuffizienz

- Prophylaxe von Nierensteinen

- verstärkte Ausleitung von Harnsäure bei gichtgefährdeten Patienten

Blasenmittel bis heute einen herausragenden Platz in der Pflanzenheilkunde. Seine Beeren und deren ätherisches Öl werden innerlich aufgrund ihrer durchspülenden und antiphlogistischen Wirkung bei Entzündungen der ableitenden Harnwege geschätzt. Auch bei dyspeptischen Beschwerden hat sich der Wacholder aufgrund seiner spasmolytischen Wirkung bewährt. Volksheilkundlich gilt er als beschützende Heilpflanze und 


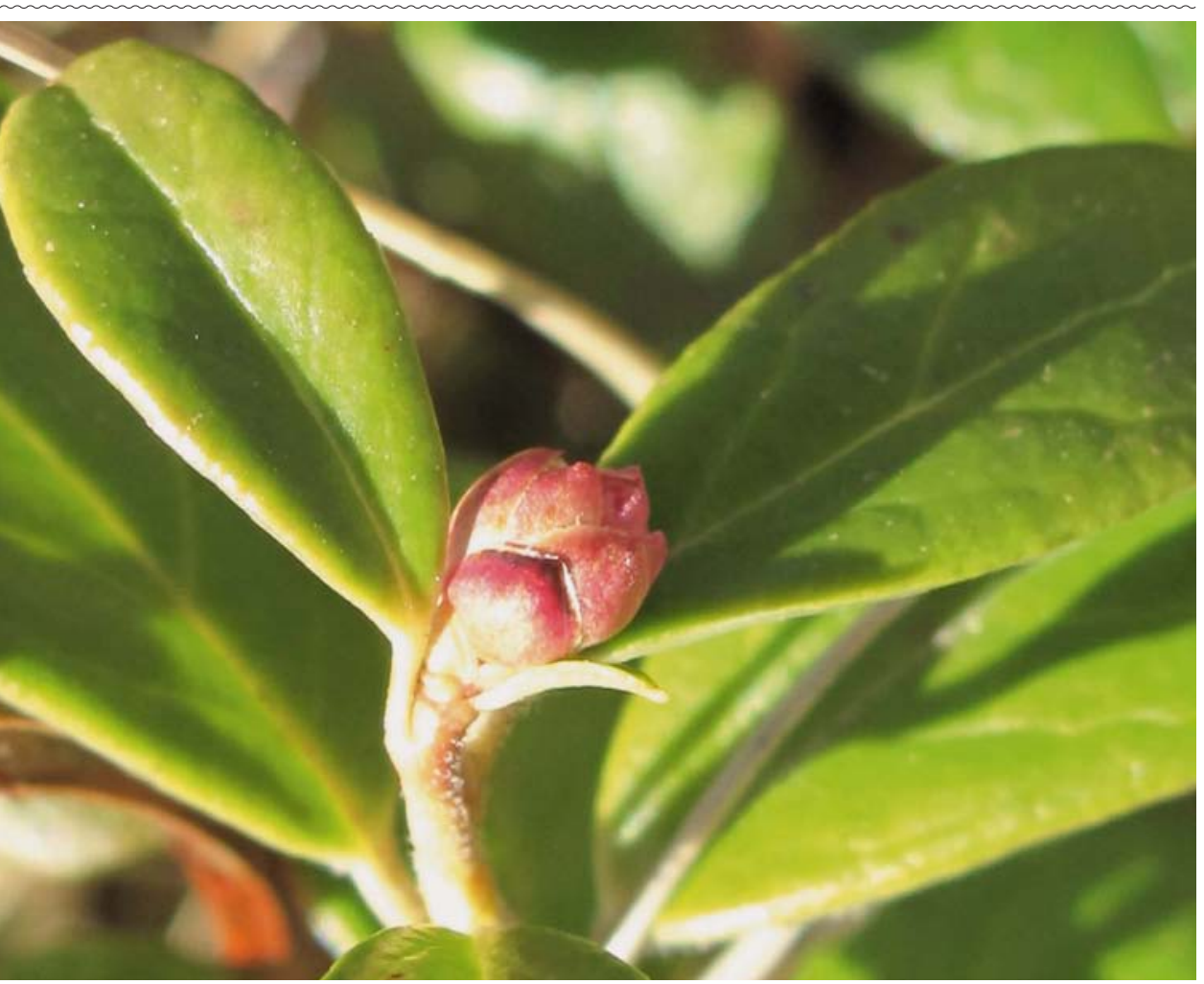

Abb. 5 Preiselbeerstrauch (Vaccinium vitis-idaea): Blütenknospe mit rötlich überlaufenden Knospenschuppen.

spielte eine wichtige Rolle in den wütenden Pestzeiten.

Die Wirkung der mit kleinen, nadeligen Schuppen bedeckten Knospen auf die Harnwege beruht insbesondere auf dem Schutz der Nierenfunktion. Sie wirken stark entzündungshemmend sowohl bei der einfachen Blasenentzündung als auch bei einer Nierenentzündung oder einer Nierenbeckenentzündung. Selbstredend gehören solche schweren Erkrankungen in die ärztliche Praxis, können jedoch durchaus mit Gemmo Wacholder unterstützt werden. Auf der Ebene der Leber wirken Wacholderknospen hepatoprotektiv und unterstützen den Leber-

- AUF EINEN BLICK

\section{Indikationen für Preisel- beer-Gemmopräparate}

- chronische Blasenentzündung, insbesondere in den Wechseljahren oder bei hormoneller Dysbalance

- grenzwertig erhöhte Harnsäurewerte im Blut (Serum) stoffwechsel sowie die Ausscheidungsund Entgiftungsfunktion.

Das Gemmopräparat Wacholder gilt bis heute als stärkstes pflanzliches Nierenmittel. Seine Wirkgebiete umfassen im Rahmen einer Begleittherapie insbesondere die tiefgreifenden, destruktiven und chronischen Erkrankungen der Niere.

\section{Preiselbeere: Frauenpflanze als Harnwegsbalsam}

Die Blätter des Preiselbeerstrauches (Vaccinium vitis-idaea) dienen in der Phytotherapie als Harnwegsdesinfiziens bei entzündlichen Erkrankungen der Harnwege. Sie enthalten den antibakteriellen Wirkstoff Arbutin in nur leicht geringerer Konzentration als die Bärentraubenblätter und schmecken in Teemischungen besser.

In der Gemmotherapie gehören Preiselbeerknospen zu den wichtigen Frauenheilmitteln. Sie kommen insbesondere bei Beschwerden im Klimakterium zur Anwendung, da die Preiselbeere den Östrogenstoffwechsel unterstützt. 


\section{- HINTERGRUND}

\section{Grundprinzipien der Gemmotherapie}

Die Gemmotherapie gilt als Teil der heutigen Phytotherapie, verwendet Knospen mit ihrem teilungsaktiven Gewebe und basiert ebenso auf sekundären Pflanzenstoffen, wenn auch in niedrigerer Konzentration. Was diese pflanzliche Therapie so besonders macht, sind jedoch zusätzliche Inhaltsstoffe, die in den fertig ausgebildenden Pflanzenteilen wie Blatt oder Blüte nicht oder kaum mehr auffindbar sind. Dazu gehören die pflanzlichen Wachstumshormone wie die Auxine und Gibberelline, aber auch viele Aminosäuren, Enzyme und andere Proteine. Auf diese Weise nutzt man wertvolle Inhaltsstoffe, die in fertig ausgebildeten Pflanzenteilen wie Blatt oder Blüte nicht oder kaum noch vorkommen. Dazu gehören pflanzliche Wachstumshormone wie Auxine und Gibberelline, aber auch viele Aminosäuren, Enzyme und andere wichtige Proteine. Erst das Zusammenwirken der Inhaltsstoffe macht die Wirkung eines Präparats aus. Nebenwirkungen und Kontraindikationen zu den im Beitrag genannten Knospenmitteln sind nicht bekannt.

Die Aufnahme der Mazerate (Kaltauszüge) aus Glyzerin und Ethanol erfolgt über die Mundschleimhaut. Dies trägt wesentlich zur Wirkung bei, denn die Proteine würden im Verdauungstrakt durch peptidspaltende Enzyme denaturiert werden und könnten so ihre volle Wirkung nicht mehr entfalten.

Der zweite Behandlungsschwerpunkt setzt am Darm an. Hier helfen die Knospenmittel bei Krämpfen und Koliken, bei (chronischer) Verstopfung wie auch beim Aufbau der Darmflora, zum Beispiel nach Gabe von Antibiotika.
Erst an dritter Stelle steht die Wirkung auf die Harnwege, die trotzdem nicht zu unterschätzen ist. Denn gerade in den Wechseljahren trocknen die Schleimhäute durch die Verminderung des Östrogens aus. Davon sind auch die auskleidenden Schleimhäute der Blase und Harnleiter betroffen. Sie bieten dadurch eine bessere Andockmöglichkeit für Bakterien. So gehören (rezidivierende) Blasenentzündungen zu den häufigeren Begleiterkrankungen des Klimakteriums.

Die Wirkung der Preiselbeerknospen auf die Harnwege beruht einerseits auf der antibakteriellen und entzündungshemmenden, andererseits auf der hormonregulierenden Wirkung. In geringerem Ausmaß unterstützen sie die Niere bei der Ausleitung der Harnsäure.

Auch wenn Preiselbeerknospen nicht zu den wichtigsten Nierenmitteln gehören, so haben sie doch ihren festen Platz in diesem Umfeld verdient. Denn so manche Blasenentzündung weiblicher Patienten im fortgeschrittenen Alter kann damit erfolgreich behandelt werden. Bei einer Blasenentzündung gibt man jede halbe Stunde zwei Sprühstöße in den Mund. Wenn sich die Symptome bessern, kann die Zeitspanne zwischen den Gaben vergrößert werden. Bei erhöhten Harnsäurewerten morgens und abends je drei Sprühstöße in den Mund geben.

Auf diese Weise kann so manche Zystitis (und Reizblase) weiblicher Patientinnen im fortgeschrittenen Alter erfolgreich behandelt werden.

Alle Bilder: ㄷ Cornelia Stern

Dieser Artikel ist online zu finden: http://dx.doi.org/10.1055/a-0935-3857

\section{Verwendete Literatur}

[1] Stern C. Gemmotherapie. Grundlagen - Indikationen - Behandlung. Stuttgart: Haug; 2018

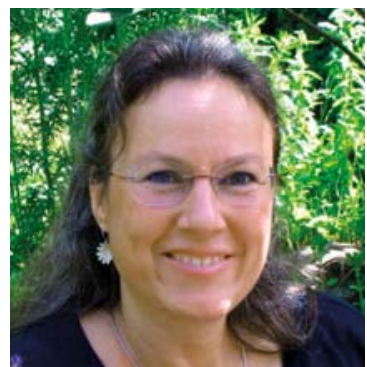

\section{Cornelia Stern}

Mitinhaberin der Freiburger Heilpflanzenschule

79111 Freiburg im Breisgau

E-Mail: cornelia.stern@heilpflanzenschule.de Internet: www.heilpflanzenschule.de

Cornelia Stern ist Apothekerin mit Spezialisierung in Naturheilkunde. Zusammen mit ihrem Mann leitet sie die Freiburger Heilpflanzenschule und ist als Dozentin für Phytotherapie, Spagyrik, Gemmotherapie und Klassische Homöopathie tätig. Zudem ist sie Autorin der Bücher „Die Heilkraft der Pflanzenknospen“ und „Gemmotherapie“ sowie Mitherausgeberin der $\mathrm{DHZ}$. 